\title{
Visualization Functionality of Virtual Factories An Enhancement to Collaborative Business Process Management
}

\author{
Ahm Shamsuzzoha ${ }^{1}$, Filipe Ferreira ${ }^{2}$, Sven Abels $^{3}$, Americo Azevedo $^{2}$ and Petri Helo ${ }^{1}$ \\ ${ }^{I}$ Department of Production, University of Vaasa, PO Box 700, Vaasa, Finland \\ ${ }^{2}$ Manufacturing Systems Engineering Unit, INESC TEC (formerly INESC Porto), Porto, Portugal \\ ${ }^{3}$ Ascora GmbH, Innovation \& Product Development, Birkenallee 43, 27777 Ganderkesee, Germany
}

Keywords: Business Collaboration, Virtual Factory, Business Process Monitoring, Process Visualization, Small and Medium Enterprise (SME).

\begin{abstract}
This paper focuses on process visualization that is applicable to managing a Virtual Factory (VF) business environment. It briefly provides all aspects of implementing the dashboard user interface that is to be used by the VF partners. The dashboard features state-of-the art business intelligence and provides data visualization, user interfaces and menus to support VF partners to execute collaborative processes. With advanced visualizations that produce quality graphics it offers a variety of information visualizations that brings the process data to life with clarity. This data visualization provides critical operational matrices (e.g. KPIs) required to manage virtual factories. Various technical aspects of this dashboard user interface portal are elaborated within the scope of this research such as installation instructions, technical requirements for the users and developers, execution and usage aspects, limitations and future works. The dashboard user interface portal presents different widgets according to the VF requirements that are to be needed to support the visualization and monitoring of various business processes within a VF. The research work highlighted in this paper is conceptualized, developed and validated within the scope of the European Commission NMP priority of the Seventh RTD Framework Programme for the ADVENTURE (ADaptive Virtual ENterprise ManufacTURing Environment) project.
\end{abstract}

\section{INTRODUCTION}

The concept of business collaboration is not new but it attracts growing interest to the business communities due to its associated benefits and the on-going globalization within the manufacturing domain. This collaboration usually begins after identifying a possible business opportunity. This business opportunity is elaborated within the possible partners, whose are selected based on specific criteria such as their capacity, skills, costs or locations. Often there exists a pool of companies with similar expertizes and products known as business community (Carneiro at al., 2010), virtual organization breeding environment (Afsarmanesh and Camarinha-Matos, 2005), industrial cluster (Flores and Molina, 2000), etc., from which potential partners are selected to form such business network, known as virtual organization, virtual enterprise, virtual factory (Jain et al., 2001), etc.

The concept of virtual factory (VF) as presented in this research provides mechanisms and tools that facilitate the creation and operation of collaborative processes in a business environment. This environment combines the power of individual factories into a single virtual factory to achieve complex manufacturing processes. It offers dynamic business portfolio like partner finding, process creation, process forecasting and optimization, information exchange as well as real-time monitoring. Along with such offers, virtual factory needs to offer proven tools or technologies that provide end-to-end integrated information and communication technology (ICT) which will help to facilitate information exchange between factories. This possibility moves beyond the boundaries of the individual enterprises involved in the business network.

In any successful business cooperation there needs to address monitoring and governance of the collaborative processes (Shamsuzzoha et al., 2013). This process monitoring enables to increase the degree of flexibility within the virtual factory 
partners and helps them to react quickly to changes and to participate in larger cross-companies manufacturing processes. The objective of this research is therefore to focus on process visualization that is applicable to monitoring and managing VF business environment. This research briefly presents all aspects of implementing a dashboard user interface that is to be used by the VF partners to process monitoring.

The rest of the paper is organized as follows: Section 2 presents theoretical framework of the research, while Section 3 states the conceptual framework of the virtual factory. Section 4 discusses the overview of VF process visualization through dashboard user interface. Section 5 illustrates a case example, where the dashboard portal is used as a tool for VF's processes management. Overall outcomes from this research are concluded with future research directions in Section 6.

\section{THEORETICAL FRAMEWORK}

To be successful in today's turbulent and competitive market environment, companies need to acquire new business models, business strategies, governance principle, processes and technological capabilities (Ermilova and Afsarmanesh, 2006). There are growing challenges towards manufacturing enterprises such as increased product variety, product's complexity and quality demands, reduced product life cycle and decreased revenue margins. In order to tackle such challenges companies, especially small and medium enterprises (SMEs) need to join efforts with others through collaboration. This collaboration offers companies to bring the potential of dynamically adjusting the needs in terms of dynamic inter-organizational models, distributed business process management, integration and coordination.

Due to recent development of ICT influences the concept of forming collaborative networks that allow manufacturing organizations to move from highly data-driven environments to more cooperative information or knowledge driven environments (Jeong and Nof, 2009; CamarinhaMatos et al., 2009). This environment develops innovative manufacturing processes and products that are capable of responding rapidly to changing/uncertain demands, demands for customtailored products and fierce international competition in the new global economy. Typical shared expertise, resources and skills through business collaboration impose companies (SMEs) high productivity levels for labor and manufacturing facilities, a high level of agility and the use of new business models to enhance the production capabilities beyond companies' borders.

Different types and forms of business collaborations are described in the literature based on the needs and perspectives. For instance virtual enterprise (VE) or virtual organization (VO), this is goal-oriented, temporary and is focused on a single project or business opportunity (Camarinha-Matos et al., 2005; Shamsuzzoha and Helo, 2012). Other type of collaboration known as collaborative networked organization (CNO), which is a network composed of largely autonomous, geographically distributed and heterogeneous in terms of operating environment, culture, social capital and goals (Camarinha-Matos and Afsarmanesh, 2006). There is specific type of collaboration network named as virtual factory (VF), which is considered as the partnerships between companies, where individual partners are connected with each other through webenabled platform and is formed and operated for designing and developing of both mass customized or one-of-a-kind product and service.

\section{VIRTUAL FACTORY: CONCEPTUAL FRAMEWORK}

The virtual factory (VF) aims at integration of multiple organizations into one virtual platform for exploiting a market opportunity. Upton and McAfee (1996) define virtual factories as "collaborative, internetworked environments in which several partners electronically share information and IS tools around a product (CAD/CAM, simulationbased design) process, or project". The VF can be implemented to ensure that manufacturing processes and sub-system designs will meet the pre-identified requirements. It is used as the source of enterprise knowledge sharing (know-how), adoption of common best practices and open source/Web-based applications are enablers to achieve both the concept of integrated enterprise and the implementation of collaborative networked enterprises for manufacturing industry.

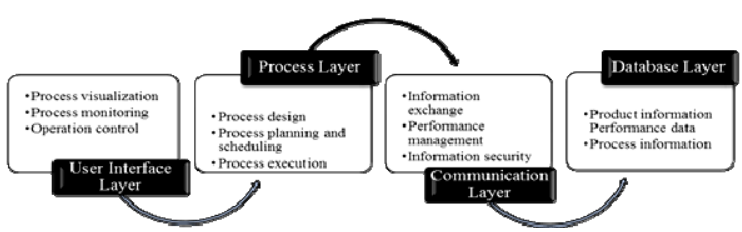

Figure 1: A conceptual architectural framework for virtual factory environment. 
The architectural framework for VF is highlighted in Figure 1. From Figure 1 it is seen that the framework consists of four different layers such as user interface layer, process layer, communication layer and database layer. Each of the layers has its own contents and functionalities. For instance, user interface layer of the VF framework responsible for process visualization, process monitoring and operation control within the VF, whereas, process layer acted upon process design, process planning and scheduling and process execution. Through the communication layer, VF partners received required information and transfer to another with secured way. All the necessary data or information is stored within the database layer of VF framework, from where requited information can be retrieved according to need.

The communication channel between VF partners' is maintained through Web-based platform. This platform is operated by the application of Internet technology that provides necessary support to exchange information between VF partners. Different VF components or layers are connected with the Web portal through porlets that allows them to interface with each other for establishing required communication channel. This channel use as the base for VF processes monitoring and management during the execution phase of the VF. Each portlet of the portal contains restrictive information depending on the predefined user role. So in the same Web portal the displayed portlets will be different for users with different roles.

\section{PROCESS VISUALIZATION THROUGH DASHBOARD USER INTERFACE}

\section{Project Context}

The global cross-industry manufacturing process (so called virtual factory) is designed using a process designer tool, where all the semantic annotations are done as well as the partners' assignment through. The partner's assignment can be based on skills, time, cost as well as environmental aspects such as $\mathrm{CO}_{2}$ emissions. The partners can be searched on the Members repository, where any company around the world can register and describe their services. Figure 2 displays the snap shot of members' repository within user interface layer. Within this repository system, all the required information such as product lines, lead-time, skills, performances, product costs, etc., of the possible virtual factory partners is stored.

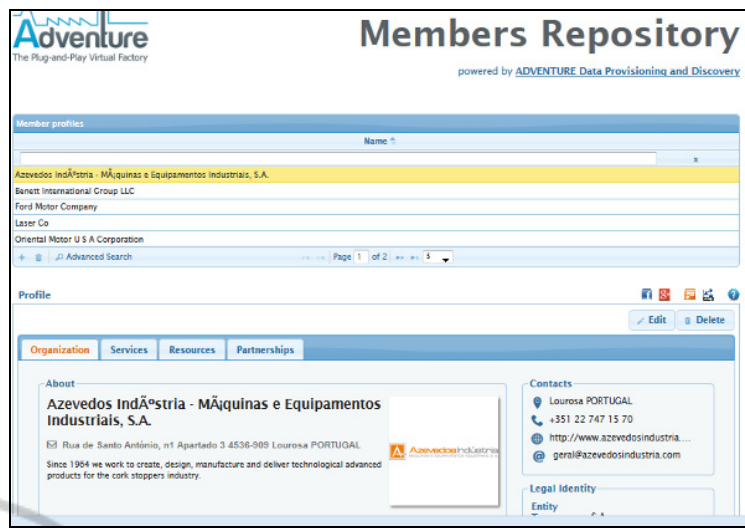

Figure 2: A snap shot of virtual factory members' repository system.

Once the global cross-industry manufacturing process is completed, the selected partners receive notifications in order to answer the service request. After all the legal aspects, the process model (BPMN2) file is translated to the Complex Process Execution Engine (CPEE) Language. The CPEE is responsible for the process execution and services invocation in the partners Legacy Systems.

\section{Process Monitoring Component Context}

A solid approach for Process Monitoring has to consider monitoring and control functionalities for the process execution, provide meaningful data in order to allow an up-to-date, real-time diagnosis of the process execution status. Process Monitoring should be able to process high amount of data and it has to be as user friendly as possible. To do so, several technologies have been evaluated and applied. Within the approach of the ADVENTURE project, data is received via Extensible Messaging and Presence Protocol (XMPP) events subscribed at the CPEE. Events and associated data are then stored at the cloud storage - a flexible and scalable data storage system, being distributed among different physical servers. A real time monitoring component shows the process flow status. A Business Intelligent Engine allows key performance indicators definition, calculation and analysis. Finally, a Rules Engine is used to create rules and trigger notifications to a holistic dashboard.

To realize the holistic dashboard view, a user based requirements elicitation has been performed with the help of user consultation in 3 different manufacturing environments: Mass Customized Engineering-To-Order (ETO), Mass Production (Make-To-Order) and Mass Production - Assembly -To-Order (ATO). Details about the user consultations may be found at the public deliverable 
D2.3 of AVENTURE at fp7-adventure.eu. - After analysing the user consultations, it became clear that the process monitoring should be organized in 3 levels:

- Level 1 - Global View, which monitors all the virtual factories that a company is participating

- Level 2 - Specific process instance level with all related data and information

- Level 3 - Specific process activity level with all related data and information

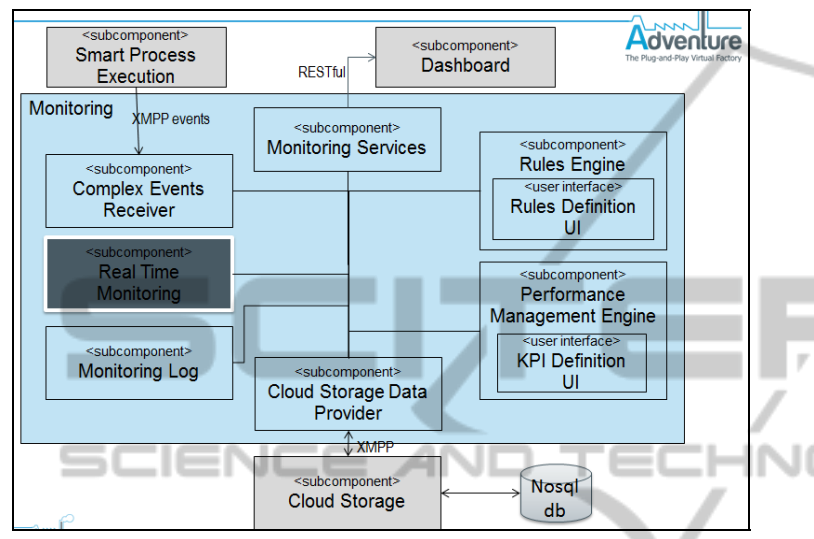

Figure 3: An architectural framework for process Monitoring Engine.

The process Monitoring Engine (Figure 3) counts with the following subcomponents:

- Events Receiver: Responsible for receiving events with raw data published by the CPEE; stores raw data in the in the Cloud Storage. XMPP protocol is applied.

- Real Time Monitoring: Show the actual status of process instances, Uses the same UI as Process Designer in order to have the same look and feel, Allows identification and tracking of the process instances.

- Monitoring Log: Queries finished process instances; Includes a search engine; Shows historical data;

- Rules Engine: Allows the definition of rules and associated actions Evaluates rules based on events and KPI values throws events and notifications to the dashboard.

- Performance Management Engine: Allow the configuration of Key Performance Indicators related to the manufacturing processes. Aggregates and analyses data; provides a graphical display in the dashboard in order to track KPIs.

- Monitoring Services Implements REST Web services; Provides Meaningful data to the other components such as the Dashboard.
Based on the data provided by the monitoring component services, a set of widgets can be included in a dashboard in order to create a holistic monitoring environment.

\section{DASHBOARD PORTAL: A CASE EXAMPLE OF PROCESSES MANAGEMENT}

The application of the dashboard user interface as designed and developed under ADVENTURE project to be used intensively to process monitoring and management. To access this user interface layer, the user needs to register his/her name and detailed in favour of his/her company within the VF. After registering in dashboard portal, the user will get a user name and password to be used to access the portal. The user roles and access rights are also can be defined by the VF broker in order to control the information flow and confidentiality among the VF partners, potential new companies, suppliers, customer and the broker. For example, the VF broker might access all the necessary information to execute the VF processes successfully, whereas VF partner might have limited access to the process information within dashboard portal and so on. Figure 4 displays the login page of the dashboard portal.

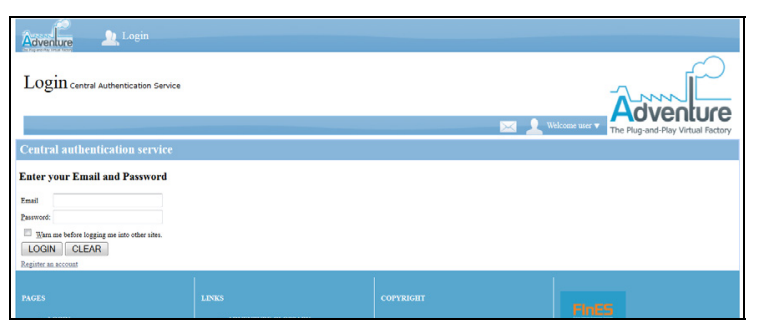

Figure 4: User login page within dashboard portal.

After successful registration and login the user can access the dashboard portal and visualize his/her updated information. Figure 5 displays the overall view of the dashboard portal. From Figure 5, it is noticed that each widget displays the required process information using different formats such as maps, graphs, texts, tables, etc. Each widget has its own functionality and levels. In order to get detailed information from each of the widgets, a user might need to navigate different levels within a widget.

This dashboard portal consists of 13 widgets such as processes, smart objects, process instances, resources, virtual production plan, alerts, message boards, etc., based on different information content 


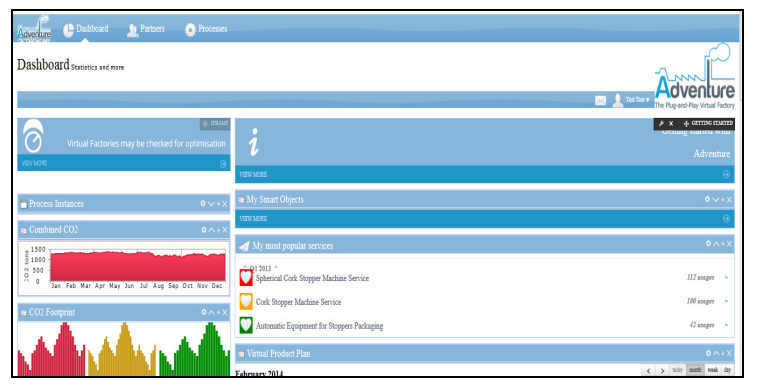

Figure 5: Overall display of dashboard user interface portal.

as required to execute the VF. For instance, processes widget contains the information related to process design, process simulation, process optimization, process adaptation, etc., as necessary to design and execute any VF process. Figure 6 displays a snap shot of processes widget within the dashboard portal. This widget contains the necessary process information such as process flow chart using BPMN (business process model and notation), all the processes used in a specific virtual factory environment (simulation, optimization, forecasting, etc.,), etc.

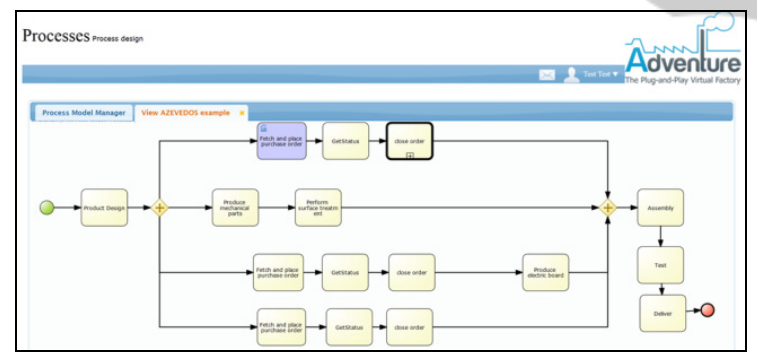

Figure 6: Processes widget snap shot within the dashboard user interface portal.

\begin{tabular}{|c|c|c|}
\hline Alerts & \multicolumn{2}{|c|}{$0 \hat{\alpha}+x$} \\
\hline Overdue tasks & 2 & ^ \\
\hline Pending tasks & 6 & ^ \\
\hline New order received & 6 & ^ \\
\hline New user registered & 2 & ^ \\
\hline Tasks over budget & 1 & ^ \\
\hline Task failure & 1 & ^ \\
\hline oh temnerature & 25 & ^ \\
\hline
\end{tabular}

Figure 7: Alerts widget snap shot within the dashboard user interface portal.
Another example snap shot of a widget named 'Alerts' can be presented as in Figure 7, where various alerts related to different VF processes are displayed. These alerts are used to get status update of different processes that are executing during the VF runtime environment. The alerts can be in the form of message such as overdue tasks, pending tasks, task failure, tasks over budgets, etc. From these status updates VF broker and/or partners are able to monitor their corresponding processes and to take necessary measures to manage the processes that are eventually contributes to minimize risks or abnormalities.

\section{CONCLUSIONS}

The virtual factory is a concept that integrates a group of manufacturing companies with the objective to achieve pre-identified business opportunity. Usually, in this type of business network companies with similar product portfolios and expertizes are collaborate with each other for sharing valuable resources and skills for mutual benefits. This collaboration cannot be successful until and unless it's associated business processes are monitored and managed properly. An effort is initiated within this research study to monitor VF business processes through dashboard user interface. This user interaction layer provides necessary technical supports to visualize the monitored data/information within VF processes.

The process monitoring component as presented in this research highlights briefly its associated subcomponents and their functionalities, which contribute to trigger an effective and efficient process monitoring. This component provides process visualization that is applicable to managing VF business environment. It focuses on meaningful monitored data and control functionalities that allow an up-to-date, real-time information repository. All the data or information is accessed as user friendly as possible and displayed over the dashboard portal.

The dashboard portal features state-of-the-art business intelligence and provides data visualization, user interfaces and menus to support VF partners to execute collaborative processes. It presents the high level process widgets as used to monitor and manage the virtual factory processes. Each of the widgets functions based on the user requirements and contains specific information repository according to its predefined functionality. All the widgets which are the integrated part of the dashboard portal execute required functionalities and visualized the 
expected process information over the dashboard. In addition to, this portal provides an easy way to visualize the status information of the VF processes in real-time business environment.

Process visualization over the dashboard portal provides critical operational metrics (e.g. key performance indicators) required to manage virtual factories. Advanced visualizations with quality graphics offer information updates that bring the process data to life with clarity. The interactive dashboard portal supports the flexibility to mould the data around unique business objectives in realtime through an intuitive graphical interface. This research highlights the necessity and reliability of a dashboard portal which is under developmental stage and will be completed in future research approach. The current dashboard portal is tested upon by using fake data, which will be validated in future with the real data from a case business network.

\section{ACKNOWLEDGEMENTS}

The authors would like to acknowledge the cofunding of the European Commission in NMP priority of the Seventh RTD Framework Programme (2007-13) for the ADVENTURE project (ADaptive Virtual ENterprise ManufacTURing Environment), Ref. 285220. The authors also acknowledge the valuable collaboration provided by the project team during the research work.

\section{REFERENCES}

Ermilova, E. and Afsarmanesh, H., 2006. Competency and profiling management in virtual organization breeding environments. Network-centric collaboration and supporting frameworks, Springer, Boston, pp.131-142.

Jeong, W. and Nof, S.Y., 2009. Collaborative e-work networks in industrial engineering. Computers \& Industrial Engineering, Vol. 57, No. 1, pp. 1-2.

Camarinha-Matos, L.M., Afsarmanesh, H., Galeano, N. and Molina, A., 2008. Collaborative networked organizations - Concepts and practice in manufacturing enterprises. Springer, New York.

Camarinha-Matos, L.M., Afsarmanesh, H. and Ollus, M., 2005. Virtual organizations - systems and practices. Springer, Boston.

Camarinha-Matos, L.M., Afsarmanesh, H., 2006. Collaborative networks: value creation in a knowledge society. Proceedings of PROLAMAT, 2006 IFIP international conference on knowledge enterprise New challenges, Springer, Shanghai, China.
Carneiro, L., Almeida, R., Azevedo, A.L., Kankaanpää, T. and Shamsuzzoha, A., 2010, An innovative framework supporting SME networks for complex product manufacturing. Proceedings of the PRO-VE 2010, Saint-Etienne, France, 10-13 October 2010.

Flores, M. and Molina, A., 2000. Virtual industry clusters: foundation to create virtual enterprises. L.M. Camarinha-Matos, H. Afsarmanesh, Heinz-H. Erbe (Eds.), Advanced in networked enterprises - Virtual organizations, balanced automation and systems integration, Kluwer Academic Publishers, Boston, pp. 111-120.

Camarinha-Matos, L.M., Afsarmanesh, H., 2005. A framework for management of $\mathrm{VO}$ breeding environment. Collaborative networks and their breeding environments, Springer, Boston.

Jain, S., Choong, N. F., Aye, K .M. and Luo, M. 2001. Virtual factory: an integrated approach to manufacturing systems modeling. International Journal of Operations \& Production Managemeent, Vol. 21, No. 5/6, pp. 594-608.

Upton, D.M. and McAfee, A.P., 1996. The real virtual factory. Harvard Business Review. July-August.

Shamsuzzoha, A., Helo, P. and Khadem, M.M.R., 2013. Smart process monitoring and management in a distributed manufacturing system: indexed towards virtual factory. Proceedings of the International Conference on Information, Operations Management and Statistics (IOMS 2013), September 1-3, Kuala Lumpur, Malaysia.

Shamsuzzoha, A. and Helo, P., 2012. Virtual enterprise management to enhance the manufacturing process collaboration. In Proceedings of the 22nd International Conference on Flexible Automation and Intelligent Manufacturing (FAIM 2012), June $10^{\text {th }}$ $13^{\text {th }}, 2012$, pp. 123-131, Helsinki, Finland. 